Marlies Y. Bongers $\cdot$ Petra Bourdrez

Jan W. van der. Steeg $\cdot$ A. Peter M. Heintz

Hans A. M. Brölmann • Ben W. J. Mol

\title{
Thermal endometrial ablation in dysfunctional uterine bleeding: an economic comparison of bipolar ablation and balloon ablation
}

Received: 21 December 2004 / Accepted: 15 March 2005/Published online: 26 August 2005

(c) Springer-Verlag Berlin / Heidelberg 2005

\begin{abstract}
Objective: A bipolar radio-frequency impedance-controlled endometrial ablation system is more effective than balloon ablation in the treatment of dysfunctional uterine bleeding. The aim of the present study was to compare the costs of both treatments, and to perform a cost-effectiveness analysis. Study design: An economic evaluation was set up alongside a randomised clinical trial comparing bipolar radio-frequency endometrial ablation and balloon ablation in 126 patients with dysfunctional uterine bleeding. Data on resources used for treatment and lost production time were prospectively collected, and costs of both treatments were calculated. Results: Mean direct medical costs per patient were $€ 1638$ for bipolar ablation and $€ 1545$ for thermal balloon ablation with a mean difference of $€ 93(95 \%$ CI $€ 45-140, P$-value 0.01$)$. Mean indirect medical costs were just over $€ 200$ in each group. Incorporation of the costs of post-ablation hysterectomies resulted in mean costs of $€ 2006$ and $€ 2053$ in the balloon group ( $P$-value 0.01$)$. In the balloon group, the cost per satisfied patient was $€ 2333$ compared to $€ 2112$ in the bipolar group. Similarly, in the bipolar group the cost per amenorrhoeic patient
\end{abstract}

M. Y. Bongers $(\varangle) \cdot$ B. W. J. Mol

Department of Obstetrics and Gynecology, Màxima Medical Center, 5500 MB Veldhoven, P.O. Box 7777, The Netherlands E-mail: my.bongers@iae.nl

Tel.: + 31-40-8888385

Fax: + 31-40-8888387

P. Bourdrez

Department of Obstetrics and Gynecology,

Vie Curie Hospital, Venlo, The Netherlands

A. P. M. Heintz

Department of Obstetrics and Gynecology,

Utrecht Medical Center, Utrecht, The Netherlands

H. A. M. Brölmann

Department of Obstetrics and Gynecology,

Free University Medical Center, Amsterdam, The Netherlands

J. W. Steeg · B. W. J. Mol

Department of Obstetrics and Gynecology, Academic Medical Center, Amsterdam, The Netherlands was $€ 4361$ and in the balloon group $€ 12831$. Conclusions: The direct costs of bipolar ablation were higher than the costs of balloon ablation. However, after inclusion of the retreatment costs, bipolar ablation was less expensive than balloon ablation.

Keywords Menorrhagia - Dysfunctional uterine bleeding $\cdot$ Endometrial ablation $\cdot$ Economic analysis · Cost-effectiveness

\section{Introduction}

Excessive menstruation is a frequent problem in premenopausal women. Menorrhagia can be caused by intracavitary abnormalities, but it also occurs in women without such abnormalities. Women with periodic uterine blood loss of $>80 \mathrm{ml}$ and a normal uterine cavity are said to have dysfunctional uterine bleeding. Ablation of the endometrium is an effective treatment in women with dysfunctional uterine bleeding [1-3].

We have previously reported on a randomised clinical trial comparing a bipolar radio-frequency impedancecontrolled endometrial ablation system and thermal balloon ablation, which are both second generation endometrial ablation devices. At one-year follow-up, amenorrhoea rates were $43 \%(34 / 83)$ in the bipolar group and $8 \%(3 / 43)$ in the balloon group (Relative Risk (RR) $0.17,95 \%$ confidence interval (CI) $0.06-0.52$ ). Moreover, 12 months after the start of treatment $90 \%$ of the patients in the bipolar group were satisfied with the result of the treatment versus $79 \%$ in the balloon group (RR 0.46, 95\% CI 0.1-1.1). We concluded that the bipolar ablation system was more effective than balloon ablation in the treatment of dysfunctional uterine bleeding.

In view of these data, we feel that the bipolar system should be the ablation treatment of choice in women with dysfunctional uterine bleeding, if the costs of this treatment are acceptable. In our randomised clinical trial, we also collected data on the use of resources for 
both treatments. The aim of the present paper was to report on the costs of both treatments, and to perform a cost-effectiveness analysis.

\section{Materials and methods}

Women with dysfunctional uterine bleeding, indicated on a pictorial chart with a Higham score of 150 points or more, were eligible for the trial [4]. The study was performed at the Máxima Medical Centre in Veldhoven, The Netherlands, between November 1st 1999 and July 1st 2001. All participants gave written informed consent before enrolment. Saline infusion sonography or diagnostic hysteroscopy were required to confirm a normal uterine cavity with histological benign endometrium and a uterine depth of between 6 and $11 \mathrm{~cm}$. All women had to have a normal pap smear, a negative chlamydia test of the cervix, and a premenopausal follicular stimulating hormone (FSH)-level of less than $40 \mathrm{IU} / 1$. Exclusion criteria were documented as coagulopathies, patients treated with anticoagulants, a desire to preserve fertility, and prior uterine surgery (except low segment caesarean section).

After a patient had given this consent, she was scheduled for surgical intervention. Randomisation was performed in the operating theatre by one of the authors (MB) just before the beginning of treatment. The randomisation sequence was computer-generated, and in order to conceal the allocation, opaque sealed envelopes were used. Patients and investigating doctors were unaware of the result of the randomisation, and remained uninformed of the ablation method used during the study. The ratio of women allocated to bipolar radiofrequency ablation to women allocated to balloon ablation was $2: 1$. The reason for the $2: 1$ ratio was to enable rapid information to be obtained on the performance of the new bipolar radio-frequency technique.

The ablation treatments were performed in both arms by one gynaecologist (MB). Patients received no medical pre-treatment, and the ablation was not timed in the menstrual cycle. All patients had Naproxen $250 \mathrm{mg} 12 \mathrm{~h}$ and $1 \mathrm{~h}$ before treatment. The methods of treatment have been described earlier (Chapter 7). In short, the bipolar endometrial ablation system consists of a generator and a disposable NovaSure device (Novacept, Palo Alto, CA, USA). The NovaSure radio-frequency generator is a constant power output generator with a maximum energy delivery of $180 \mathrm{~W}$. The device consists of a single use, conformable bipolar electrode mesh, mounted on an expandable frame that can create a confluent lesion involving the entire interior surface area, within the cavity of the uterus.

The balloon ablation system (ThermaChoice I Gynecare, Somerville, NJ, USA) consists of a generator and a balloon catheter [5]. The balloon is inserted into the uterine cavity. A thermistor in the balloon is used to raise the temperature of the fluid to $87{ }^{\circ} \mathrm{C}\left(170{ }^{\circ} \mathrm{F}\right)$ for a period of $8 \mathrm{~min}$, while maintaining the pressure of the balloon at $170 \mathrm{mmHg}$ or higher. Endometrial thinning was performed by aspiration curettage prior to the balloon treatment procedure [6].

Follow-up visits were carried out at the outpatient clinic at three, six and 12 month intervals after the initial treatment. At these, the patients were seen by a doctor who was unaware of the treatment that had been performed. At each visit, the presence of amenorrhoea was registered, and patients expressed their satisfaction with the treatment result. Levels of satisfaction were categorised as completely satisfied, satisfied, doubtfully satisfied or not satisfied. Furthermore, it was noted as to whether a reintervention had been performed, or whether a patient had started using oral contraceptives.

After treatment of 44 patients, a technical failure in the bipolar generator was discovered. No analysis of results was performed until the reason for the error was established. The precise moment at which the error occurred was not known, although it was clear that during the first five bipolar radio-frequency treatment procedures, the generator had worked properly.

\section{Costs}

The mean costs of each treatment were calculated by multiplying used resources and resource unit prices. Standardised unit costs were calculated for the Máxima medical centre. A distinction was made between costs of medical interventions (direct costs) and costs resulting from productivity losses (indirect or time costs) [7]. Resource utilisation was assessed using individual data in the case record forms. Resources counted were: duration of surgical procedure, days of day-care, extra hospital stay from the moment of randomisation in days, visits to the outpatient clinic, repeat ablation and hysterectomy. Each patient was sent a questionnaire concerning professional and non-professional domiciliary care, transportation costs, use of sanitary towels, visits to the general practitioner, and productivity loss. Trial specific resource utilization and associated costs were excluded from the analysis.

Resource unit prices reflected: unit costs for staff, materials, equipment, housing, depreciation, and overheads, the latter both at department level and at hospital level. Unit prices were calculated for all medical resources accounted for. Since in the Dutch health care system the hospitals bill the patient's insurance company and they are managed on a non-profit basis, the calculated costs are an appropriate measure of the societal cost of direct medical care.

Prices were calculated and reported in European Euros $(€)$. Since all costs were realised approximately 12 months after treatment, a correction for differential timing of economic costs was not performed. Direct costs were calculated using data from all of the patients included in the trial, whereas calculation of total costs was limited to those patients who completed the questionnaire. The CI around the mean costs of each treatment and around the difference in costs were obtained 
using a bootstrap sampling procedure [8]. For this purpose, 2000 random samples with replacement were drawn from the distribution of total costs in the two treatment groups.

\section{Cost-effectiveness}

In the cost-effectiveness analysis, we used satisfaction with treatment result and the number of amenorrhoeic patients as measures of the effectiveness of the ablative treatments. We calculated the cost per patient satisfied with the treatment result after one year, and the cost per amenorrhoeic patient after one year.

\section{Sensitivity analysis}

Sensitivity analysis was performed to explore the effect of plausible changes in key variables on the results of the cost analysis. Key variables considered were: effectiveness of the bipolar treatment, cost of the bipolar device and the time the generator had been in use, the number of hysterectomies due to dissatisfaction with the treatment result, and the number of ablative procedures performed per year at the centre. The latter factor might be important, since the high cost of the hardware might affect the cost per treatment if the number of procedures per year is relatively low.

In view of the technical failure in the bipolar generator (discovered after inclusion of 44 patients), we also performed a cost-effectiveness analysis in which we used data from the patients that were randomised after the failure was corrected.

\section{Results}

Patients

Between November 1st 1999 and July 1st 2001, 126 women were included in the trial, of which 83 were allocated to the bipolar group, and 43 to the balloon group. Table 1 shows the baseline characteristics of the two groups. There were more patients with a retroverted uterus in the bipolar group (16\% vs. $9 \%$ ), but otherwise the two groups were comparable.

One patient that had already been randomised to the bipolar treatment panicked in the operating room and refrained from the ablative treatment. One year later she had not been treated. Her menstrual blood loss was still heavy but she wanted no further treatment. There were no complications during treatment in both arms of the study. Four patients had a hysterectomy in the bipolar group and four in the balloon group (RR $0.47,95 \% \mathrm{CI}$ 0.07-3.3).

Resource units used for each treatment and their prices are presented in Table 2. Whilst the mean duration of the bipolar procedure was $9.0 \mathrm{~min}$ (range 5-32 min), treatment with the balloon procedure lasted $14 \mathrm{~min}$ on average (range 9-40-min). In both groups, one patient had to be admitted to the hospital overnight due to pain and nausea after the treatment. In the bipolar group there were three patients in whom two disposable devices were required, whereas this was the case in one patient in the balloon group.

The questionnaire on domiciliary care and productivity loss was returned by 83 patients $(66 \%)$. There were no significant differences between responders and nonresponders with respect to baseline characteristics or treatment allocation between the two groups. In the group allocated to bipolar ablation, 16 patients $(20 \%)$ had domiciliary care from friends or family after one year, whereas in the group allocated to thermal balloon ablation, four patients $(9 \%)$ had domiciliary care from friends or family. The mean duration of help was $6.6 \mathrm{~h}$ in the bipolar group versus $4.9 \mathrm{~h}$ in the balloon group. Out of the 52 responders in the bipolar group, there were $34(66 \%)$ who used sanitary towels in the first year after treatment, as compared to 28 out of $31(90 \%)$ in the balloon group.

The costs of the bipolar device were $€ 850$ per piece, as compared to $€ 735$ in the balloon group. The costs of the

Table 1 Baseline characteristics

\begin{tabular}{lll}
\hline & Bipolar group $(N=83)$ & Balloon group $(N=43)$ \\
\hline Age (years) (mean, SD) & $42.6(4.9)$ & $43.1(3.8)$ \\
Duration of menstruation (days) (mean, SD) & $7.7(2.5)$ & $8.1(2.2)$ \\
Number of patients with clots (\%) & $76(92 \%)$ & $38(88 \%)$ \\
Duration of clots (days) (mean, SD) & $3.5(2.0)$ & $3.3(2.0)$ \\
Pictorial chart (median, min, max) & $515(150-3401)$ & $660(188-3220)$ \\
Dysmenorrhoea & & \\
Moderate & $17(21 \%)$ & $13(30 \%)$ \\
Severe & $34(41 \%)$ & $16(37 \%)$ \\
Uterus & & \\
Anteverted & $49(60 \%)$ & $33(77 \%)$ \\
Midposition & $10(12 \%)$ & $4(9 \%)$ \\
Retroverted & $13(16 \%)$ & $4(9 \%)$ \\
Missing & $10(12 \%)$ & $2(5 \%)$ \\
Haemoglobin (mmol/L) (mean, SD) & $8.0(0.83)$ & $7.9(0.90)$ \\
FSH (IU/L) (mean, SD) & $6.5(3.5)$ & $6.1(4.3)$
\end{tabular}


Table 2 Average use of resources needed for bipolar ablation and thermal balloon ablation

\begin{tabular}{|c|c|c|c|c|c|}
\hline & $\begin{array}{l}\text { Unit price } \\
\text { (Euro) }\end{array}$ & $\begin{array}{l}\text { Bipolar group } \\
(n=83)\end{array}$ & $\begin{array}{l}\text { Costs for } \\
\text { bipolar group } \\
\text { (Euro) }\end{array}$ & $\begin{array}{l}\text { Balloon } \\
\text { group } \\
(n=43)\end{array}$ & $\begin{array}{l}\text { Costs for } \\
\text { balloon } \\
\text { group (Euro) }\end{array}$ \\
\hline Duration of theatre time & $8.7 \min ^{-1}$ & $30.9^{a}$ & 259.4 & $36.4^{a}$ & 319.2 \\
\hline Cost of gynaecologist & & & 98 & & 98 \\
\hline Device (hardware) & $15,000 / 8000$ & & 60 & & 32 \\
\hline Device (disposable) & $850 / 735$ & $86^{b}$ & 880.7 & $44^{b}$ & 752.1 \\
\hline Outpatient visits & 29 & 1.24 & 36.0 & 1.33 & 38.4 \\
\hline Direct costs & & & 1638 & & 1545 \\
\hline Data from questionnaire & & $(n=52)$ & & $(n=31)$ & \\
\hline Sanitary towels & 5.0 per month & $34(66 \%)$ & 19.7 & $28(90 \%)$ & 33.5 \\
\hline Lost labour procedure (mean number of days) & 64 & 1.0 & 63.9 & 1.2 & 78.0 \\
\hline Lost labour outpatient visit & 20 & 1.24 & 24.8 & 1.33 & 26.5 \\
\hline & & & 2006 & & 2053 \\
\hline
\end{tabular}

${ }^{a}$ Operation time in minutes ${ }^{b}$ Absolute numbers ${ }^{c}$ Price per day professional domiciliary care ${ }^{d}$ Including $€ 10$ transportation costs. Values are mean numbers unless stated otherwise

hardware for the bipolar system were $€ 15,000$, versus $€ 8000$ for the balloon system. With 50 ablative procedures per year, and a system lifetime of five years, this gives a cost of $€ 60$ per treatment in the bipolar group and $€ 32$ in the balloon group. The costs per lost day of labour were set at $€ 64$ [9].

The mean direct costs per patient allocated to the bipolar group were $€ 1638$ (Table 2). The mean direct costs in patients allocated to balloon ablation were $€ 1545$. The mean difference between both groups was $€ 93$ (95\% CI €45-€140, $P$-value 0.01$)$. The mean indirect costs were $€ 225$ and $€ 229$, respectively ( $P$-value 0.91 ). The mean total costs for the bipolar group were $€ 1863$ and for the balloon group $€ 1774$.

The amenorrhoea rates were $43 \%$ in the bipolar group and $8 \%$ in the balloon group. This resulted in a cost-effectiveness ratio of $€ 4335$ per amenorrhoeic patient in the bipolar group versus $€ 22,175$ in the balloon group (Table 3). The satisfaction rates of $90 \%$ in the bipolar group and $79 \%$ in the balloon group resulted in cost-effectiveness ratios of $€ 1820$ per satisfied patient in the bipolar group versus $€ 1956$ per satisfied patient in the balloon group (Table 3). Sensitivity analysis did not alter the outcome of the analysis.

At the one-year follow-up, four patients in each group underwent a hysterectomy. Due to this interventions, the mean total cost per patient increased to $€ 2006$ in the bipolar group and $€ 2053$ in the balloon group, whereas the number of satisfied patients rose to $95 \%$ in the bipolar group and $88 \%$ in the balloon group. The cost-effectiveness ratios became $€ 2112$ in the bipolar group and $€ 2333$ in the balloon group.

After excluding the 44 patients that were included before the defect in the bipolar generator was detected, the mean total costs were $€ 1845$ in the bipolar group versus $€ 1774$ in the balloon group, a marginal change compared to the costs for the whole group. After excluding the bipolar procedures performed with the defective bipolar generator, the satisfaction rates in the bipolar group increased to $94 \%$ and the amenorrhoeic patients to $55 \%$. In the bipolar group, the costs per amenorrhoeic patient dropped to $€ 1972$, whereas the costs per satisfied patient dropped to $€ 1371$.

\section{Discussion}

This study compared the costs of a bipolar endometrial ablation system with those of balloon ablation in the treatment of dysfunctional uterine bleeding. We found statistically significantly higher direct and total costs after the one year follow-up for the bipolar ablation compared to the balloon ablation, although the economic relevance of the difference was only marginal, with this difference being only $€ 88$ for the total cost. If the hysterectomy patients were included in the calculation, the costs of the balloon ablation were higher than the bipolar ablation. With respect to amenorrhoea and with respect to patient satisfaction, the cost-effectiveness was significantly better in the bipolar group compared to the balloon group.

In the Dutch health care system, costs of health care are paid by insurance companies (private or government-owned companies). The hospitals provide care to the patients and subsequently bill the insurance company. However, since the billing statements are not based on actual cost calculations, they do not represent real costs. Therefore, we calculated the real costs that had to be made to perform endometrial ablation in patients with dysfunctional uterine bleeding. 
Table 3 Cost-effectiveness of ablative treatments
Bipolar ablation

Balloon ablation

Result of initial treatment

Amenorrhoeic patients

Satisfied patients

Direct medical costs

Direct medical costs per amenorrhoeic patient

Direct medical costs per satisfied patient

Total costs

Total costs per amenorrhoeic patient

Total costs per satisfied patient

Result of treatment strategy in the first year Amenorrhoeic patients (including hysterectomy)

Satisfied patients (including hysterectomy)

Costs after one year (including hysterectomy)

Total costs per amenorrhoeic patient

Total costs per satisfied patient

$\begin{array}{ll}43 \% & 8 \% \\ 90 \% & 79 \% \\ 1.638 & 1.545 \\ 3.809 & 19.313 \\ 1.820 & 1.956 \\ 1.862 & 1.774 \\ 4.335 & 22.175 \\ 2.071 & 2.246 \\ & \\ 46 \% & 16 \% \\ 95 \% & 88 \% \\ 2.006 & 2.053 \\ 4.361 & 12.831 \\ 2.112 & 2.333\end{array}$

One of the cost variables considered was the number of ablative procedures performed per year at the centre. 50 ablations a year seems a realistic estimate. The costs of a specific ablation depend on the costs of the device and on the costs of the generator. The lifetime of the generator might be important, since the high costs of the hardware might affect the costs per treatment when the number of procedures per year is relatively low. We assumed that the generator would be in use for five years, which resulted in hardware costs for the bipolar device of $€ 60$, compared to $€ 32$ in the balloon group. Thus, these relative low hardware costs of the devices will result in only a limited impact from the number of procedures per year on the cost-effectiveness. Varying the number of procedures per year to 25 or 100 did not alter the outcome of the analysis.

The cost of the operating room was derived from the costs of anaesthesiologists and operating room staff, overhead costs, management staff, operation room housing, and number of days used. A mean bipolar procedure lasted 9 min compared to $14 \mathrm{~min}$ for a balloon procedure. The time needed for anaesthesia, to prepare patients and to change patients was fixed at $22 \mathrm{~min}$. As a consequence, eight bipolar procedures can be performed in $4 \mathrm{~h}$ operating time, as compared to 6.5 balloon ablations. This resulted in costs of $€ 259$ for a bipolar ablation and $€ 319$ for a balloon ablation. Other authors have reported a procedure time for bipolar procedure of $4.2 \mathrm{~min}$, which is even shorter than the $9 \mathrm{~min}$ reported in the present study [3]. This can led to an extra reduction in the cost of the bipolar procedure.

Our study was limited to cost of the initial treatment and to a relatively short period of follow-up of 12 months. Cost of reintervention is an important additional issue in the economic evaluation of the treatment of dysfunctional uterine bleeding. For instance, in the comparison between bipolar ablation and balloon ablation, bipolar ablation had a significant higher satisfaction and amenorrhoea rate compared to balloon ablation, which may result in a lower reintervention rate, thus making the bipolar method a less expensive strategy. A longer follow-up will be needed to shed further light on reintervention rates and additional costs in both strategies.

Previous studies have suggested that ablation often reduces costs considerably compared to hysterectomy [10-13]. The key long-term resource cost, however, is the retreatment rate of women in the ablation group. In the Bristol randomised trial that compared endometrial resection versus hysterectomy, the total costs of resection were $53 \%$ of the costs of hysterectomy after four months, whereas that percentage had increased to $71 \%$ at a mean follow-up of 2.2 years $[12,13]$. An important issue is whether this cost gap will narrow further, but previous studies have demonstrated that retreatment rates do not increase significantly two years after the initial procedure [1]. It is likely that the cost advantage of endometrial ablation over (abdominal) hysterectomy will remain, whatever the period of follow-up.

However, the crucial issue to consider is the relative cost-effectiveness of treatments of dysfunctional uterine bleeding, which requires not only an assessment of costs but also of benefits. Therefore, we calculated the costs of bipolar and balloon ablation in relation to amenorrhoea and satisfaction. Figure la shows the costs of both strategies compared to the percentage of patients with amenorrhoea at one year of follow-up. Figure $1 \mathrm{~b}$ also shows the costs for both strategies, but instead compared to the percentage of satisfied patients.

In Fig. 1a and b, we also incorporated data from the study of Hurskainen et al [14], who has previously reported on the cost-effectiveness of the levonorgestrelreleasing IUD and hysterectomy in the treatment of menorrhagia. To facilitate this comparison, we used the lower estimate of productivity loss reported in the Finnish study (for the IUD group $€ 1227$ and for the hysterectomy group $€ 3067)$.

From Fig. 1a, it can be seen that the percentage of patients with amenorrhoea after one year is comparable in the IUD strategy and the bipolar ablation groups. The balloon ablation is inferior to the other two strategies, with a far lower percentage of patients being amenorrhoeic at a cost similar to that of the bipolar 


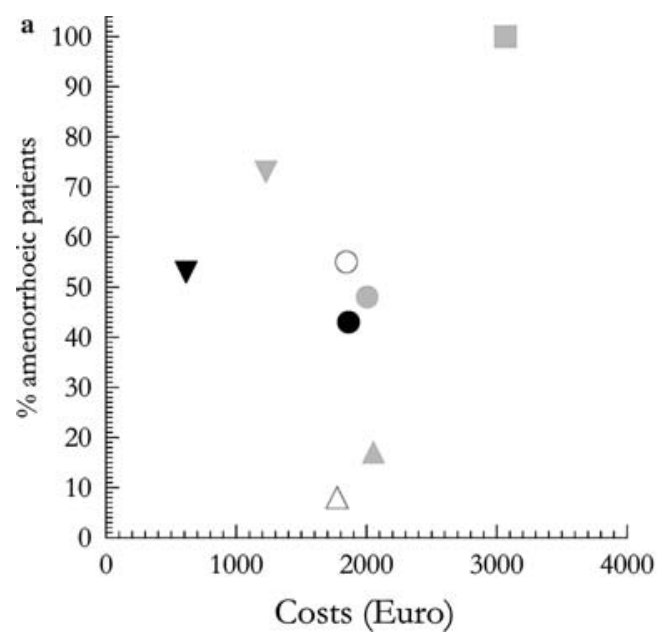

Fig. 1a-b Cost-effectiveness of the evaluated strategies (in terms of amenorrhoea, a, and satisfaction, b), together with the costeffectiveness of hysterectomy and a levonorgestrel-releasing device.

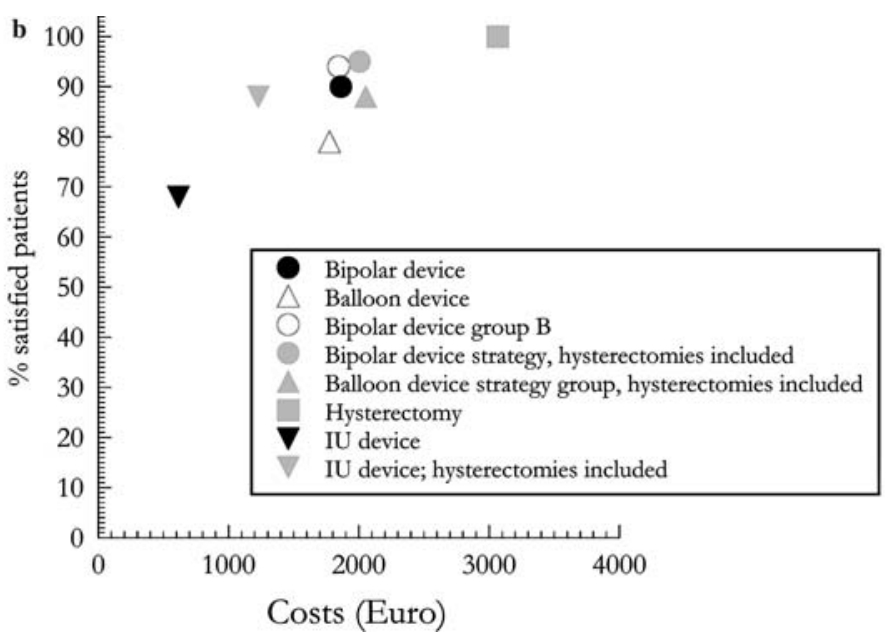

Data on the two latter treatments were obtained from Hurskainen et al [14]. Bipolar B group consist of patients who were randomised after the failure with the NovaSure generator had been corrected ablation. Figure $1 \mathrm{~b}$ shows that the satisfaction rates after bipolar ablation were slightly better at 12 months follow-up than the satisfaction rates after balloon ablation, and that the cost-effectiveness ratios were comparable. A satisfaction rate was not reported in the Finnish study. We assumed that the $68 \%$ of the patients who had their IUD in situ at one year of follow-up were satisfied with their treatment, whereas the remaining $32 \%$ were not. Taking into account the cost of the hysterectomies that were performed in $20 \%$ of the patients in the IUD group, the cost-effectiveness ratio became comparable to that of the ablative strategies reported in our study. Figure $1 \mathrm{a}$ and $\mathrm{b}$ also show that hysterectomy is not cost-effective as a first choice treatment compared to either a levonorgestrel-releasing device or thermal ablation.

In the Finnish study, no distinction was made between vaginal, abdominal, or laparoscopic hysterectomy. The vaginal hysterectomy reduces hospital stay compared to abdominal hysterectomy, whereas the duration of the surgical procedure of a vaginal hysterectomy is shorter than the duration of a laparoscopically-assisted hysterectomy (LAVH) [15]. An economic evaluation comparing LAVH and abdominal hysterectomy showed LAVH to be significantly more expensive [16]. These two studies support the conclusion that the vaginal hysterectomy is the most cost-effective route for hysterectomy. As far as we know, a comparison of the cost of vaginal hysterectomy with those of abdominal hysterectomy and LAVH has not been performed. Theoretically, an uncomplicated vaginal hysterectomy can result in a day-care hospital admittance and will substantially reduce the costs. However, Fig. 1b shows that hysterectomy will be cost-effective if this major operation does not cost more than $€ 2000$.

The potential for second generation ablation techniques to be performed under local anaesthesia can generate a considerable cost advantage. This approach will save costs, because an expensive operating theatre, with its equipment and trained staff, is not necessary at the outpatient clinic. The operation time for bipolar radio-frequency endometrial ablation was almost half that of the balloon procedure. This may be an important advantage in an outpatient setting, even though a cervical dilation of up to $7.5 \mathrm{~mm}$ is necessary to perform the bipolar technique, and no cervical dilation is needed for balloon ablation. Balloon ablation has been performed under local anaesthesia [17]. Only $61 \%$ of the patients would be happy to undertake the procedure again under local anaesthesia, which is a rather low percentage. Future research on the acceptability and costs of bipolar ablation as an outpatient procedure should be performed.

In conclusion, the direct costs of bipolar ablation were higher than balloon ablation. However, when the costs of retreatment were factored in, the bipolar ablation was found to be less expensive than the balloon ablation. Subgroup analysis showed higher cost-effectiveness per satisfied and amenorrhoeic patient for the bipolar group than for the balloon group.

\section{References}

1. Aberdeen Endometrial Ablation Trials Group (1999) A randomised trial of endometrial ablation versus hysterectomy for the treatment of dysfunctional uterine bleeding: outcome at four years. Br J Obstet Gynaecol 106:360-366

2. Loffer FD (2001) Three-year comparison of thermal balloon and rollerball ablation in treatment of menorrhagia. $\mathbf{J}$ Am Assoc Gynecol Laparose 8:48-54

3. Cooper J, Gimpelson R, Laberge P, Galen D, Garza-Leal JG, Scott $\mathbf{J}$ et al (2002) A randomized, multicenter trial of safety and efficacy of the NovaSure system in the treatment of menorrhagia. J Am Assoc Gynecol Laparosc 9:418-428

4. Higham JM, O'Brien PMS, Shaw RW (1990) Assessment of menstrual blood loss using a pictorial chart. Br J Obstet Gynaecol 97:734-739 
5. Neuwirth RS, Duran A, Singer A, MacDonald R, Bolduc L (1994) The endometrial ablator: a new instrument. Obstet Gynecol 83:792-796

6. Amso NN, Stabinsky SA, McFaul P, Blanc B, Pendley L, Neuwirth R (1998) Uterine thermal balloon therapy for the treatment of menorrhagia: the first 300 patients from a multicenter study. Br J Obstet Gynaecol 105:517-523

7. Drummond MF, Stoddart GL, Torrance GW (1986) Methods for the economic evaluation of health care programs. Oxford University Press, Oxford

8. Gold MR, Siegel JE, Russell LB, Weinstein MC (1996) Costeffectiveness in health and medicine. Oxford University Press, Oxford, New York, pp 259, 262-263

9. Koopmanschap MA, Van Ineveld BM (1992) Towards a new approach for estimating indirect costs of disease. Soc Sci Med 34:1005-1010

10. Cameron I, Mollison J, Pinion S, Atherton-Naji A, Buckingham K, Torgerson D (1996) A cost comparison of hysterectomy and hysteroscopic surgery for the treatment of menorrhagia. Eur J Obstet Gynecol Reprod Biol 70:87-92

11. Brooks P, Clouse J, Stockwell Morris L (1994) Hysterectomy versus resectoscopic endometrial ablation for the control of abnormal uterine bleeding. A cost comparative study. J Reprod Med 39:755-760
12. Dwyer N, Hutton J, Stirrat GM (1993) Randomised controlled trial comparing endometrial resection with abdominal hysterectomy for the surgical treatment of menorraghia. Br J Obstet Gynaecol 100:237-243

13. Sculpher M, Dwyer N, Byford S, Stirrat G (1996) Randomised trial comparing hysterectomy and transcervical endometrial resection: effect on health related quality of life and costs two years after surgery. Br J Obstet Gynaecol 103:142-149

14. Hurskainen R, Teperi J, Rissanen P, Aalto AM, Grenman S, Kivela A, Kujansuu E,Vuorma I, Yliskoski M, Paavonen J (2001) Quality of life and cost-effectiveness of levonorgestrelreleasing intrauterine system versus hysterectomy for treatment of menorrhagia: a randomised trial. Lancet 357:273-277

15. Ottosen C, Lingman G, Ottosen L (2000) Three methods for hysterectomy: a randomised, prospective study of short term outcome. Br J Obstet Gynaecol 107:1380-1385

16. Lumsden M, Twaddle S, Hawthorn R, Traynor I, Gilmore D, Davis $\mathbf{J}$ et al (2000) A randomised comparison and economic evaluation of laparoscopic-assisted hysterectomy and abdominal hysterectomy. Br J Obstet Gynaecol 107:1386-1391

17. Byrd L, Chia K (2002) Balloon ablation: is this an outpatient procedure? J Obstet Gynaecol 22:205-208 